\title{
A Case Study on Research in Engineering Education: Designing, Testing, and Administering the PACES-2 Survey on Academic Integrity
}

\author{
Cynthia J. Finelli ${ }^{1}$, Jamie L. Szwalek ${ }^{2}$, Donald D. Carpenter ${ }^{3}$, and Trevor S. Harding ${ }^{4}$
}

\begin{abstract}
Most engineering educators excel at planning and conducting technical research in their field, but few are proficient doing this for a project in engineering education. Recently, however, there has been increased emphasis on conducting rigorous research in engineering education. This paper provides practical advice for planning and conducting such research. The authors use their long term project to predict academic dishonesty in engineering college students as a case study representing one approach to research in engineering education. In particular, the authors present the design, testing, and administration of a two-part survey instrument to collect information from college students about their decisions related to cheating.
\end{abstract}

Index Terms-academic integrity, cheating, research in engineering education, survey development.

\section{INTRODUCTION}

Research in engineering education is becoming increasingly common at schools and colleges of engineering, yet few resources address practical strategies and guidelines for conducting research in the area. Olds, Moskal, and Miller [17] assert that successful research in engineering education involves the application of methodologies that have been derived from higher education (i.e., surveys, interviews, focus groups, conversational analysis, and observation) to study phenomena related to teaching and learning in the field of engineering. However, engineering faculty are often unfamiliar with such methodologies and are overwhelmed by challenges that include knowing how to frame a meaningful study, designing effective research instruments, ensuring that the instruments are valid and reliable, and analyzing the resulting data [19]. Further, because research in engineering education may involve collecting data from human subjects, many new challenges arise: the research instruments must be carefully worded, directions must be clear, informed consent must be obtained, and other policies pertaining to human subjects' research must be followed. Research already completed in the field of education provides a systematic approach that the engineering education community may use to guide its own assessment and evaluation efforts [17]. This paper highlights the PACES Team's research as a case study.

The PACES Team (Finelli, Carpenter, and Harding) is a group of engineering faculty and administrators with a common interest in understanding the perceptions and attitudes about cheating among engineering undergraduate students (i.e., P.A.C.E.S.). Research indicates that the percentage of undergraduates who self-report cheating differs by college major $[4,6,7,11,12,13,14,20]$ and that engineering students self-report some of the highest rates of cheating among students in all disciplines. The underlying goal of the PACES Team is to develop a better understanding of these findings and identify ways to improve the integrity of engineering professionals. Since the team began its collaborative efforts in the year 2000, it has studied the relationship between college cheating and unethical behavior in the workplace for engineering undergraduates and has identified factors (including perceptions and attitudes about cheating) that influence engineering student decisions.

Although no member of the team was formally trained in methods for educational research, the team has sought advice from experts in the field, has developed the ability to plan and conduct research in engineering education, and has overcome many of the challenges outlined above. This paper provides advice from the team's perspective, as engineering educators conducting educational research. In particular the authors describe their approach for designing, testing, and administering a two-part survey instrument to collect information from college students about their decisions related to cheating.

\section{DESIGNING THE RESEARCH STUdY}

The research process for engineering education is analogous to that in other fields (Figure 1): select a general research problem, review the literature (and identify a theoretical framework in which to describe the problem), select a specific research problem(s) or question(s), collect data, analyze and present the data, and finally interpret the findings and state the conclusions and/or generalizations about the problem [14]. While the research provides insight to important questions in the field, the ultimate goal is to disclose the information and

\footnotetext{
Cynthia J. Finelli, Managing Director of Center for Research on Learning and Teaching North and Associate Research Scientist of Undergraduate Engineering Education, University of Michigan, cfinelli@umich.edu

2 Jamie L. Szwalek, Graduate Student of Naval Architecture and Marine Engineering, University of Michigan, jlmiller@umich.edu

3 Donald D. Carpenter, Assistant Professor of Civil Engineering, Lawrence Technological University, dcarpenter@1tu.edu

4 Trevor S. Harding, Associate Professor of Manufacturing Engineering, Kettering University, tharding@kettering.edu 
determine to what extent the data can be generalized and synthesized with existing research. This is significant in that the results allow for methods to be derived, used, and evaluated in the field which may lead to better educational practices.

\section{Identifying Theoretical Frameworks and Research Questions}

In disciplinary research, it is important to identify a worthwhile problem that is of significance to the research community, and the same concept holds true in the field of engineering education. As such, choosing a theoretical framework in which to discuss the problem and formulating appropriate research questions are important steps in designing the research study. Radcliffe and Jolly [19] suggest that "... the framework should be constructed from the principles of demonstrable practicality in order for the research to gain credibility and be widely accepted." This implies that any study should be based on an authentic educational task and, if successful, should achieve results that significantly impact learning and/or teaching practice. Gall, Gall, and Borg [10] assert that "... a theoretical framework allows researchers to identify commonalities in otherwise isolated phenomena" and "...the laws of a theory enable researchers to make predictions and to control phenomena." The process of identifying a suitable framework requires a comprehensive review of the literature (i.e., journals for educational research, higher education, applied social psychology, social sciences, anthropology, etc.). The process is often iterative and time-intensive, and it may result in adapting an existing theory or modifying one to suit the needs of the research.

Using the theoretical framework to structure the problem, the next step is to pose specific research questions that can guide the work and allow for meaningful interpretation of the results. Identifying appropriate research questions can involve testing a hypothesis (commonly termed theory-based research), extending previous work, establishing the validity of research findings across different populations, identifying trends or changes over time, confirming important findings using different methodologies, or developing more effective or efficient interventions [10]. Creswell [7] suggests that the questions be open-ended, evolving, and nondirectional; that they restate the purpose of the study in more specific terms; that they start with words such as "what" or "how" (such as "What factors influence college students' decisions to cheat?" rather than "why" such as "Why do college students cheat?"); and that they number five to seven. When posing the questions, it is also important that they be subject to empirical investigation and that they be congruent with the desired outcomes of the research [15].

PACES Research-A Case Study: For the PACES-2 Project, the team began with an interest in understanding more about factors that influence students' decisions about cheating in college. The PACES Team explored several theoretical frameworks about decision making and adopted Ajzen's Theory of Planned Behavior [1] from the applied social psychology field as a model for decisions made by college students about cheating. Because research has suggested that an additional moral component might be critical to understanding decisions about cheating [2, 5], the team included an additional variable to the model, thus modifying an existing theory. Using the framework, the team posed several research questions including: (1) "Can the model be appropriately applied to college students' decisions about cheating?" (2) "How does the inclusion of a moral component variable affect the model?" (3) "How do the variables of the model differ by discipline?" and (4) "What are the effects of class level on the relative significance of variables of the model?" The project was designed to address this series of questions.

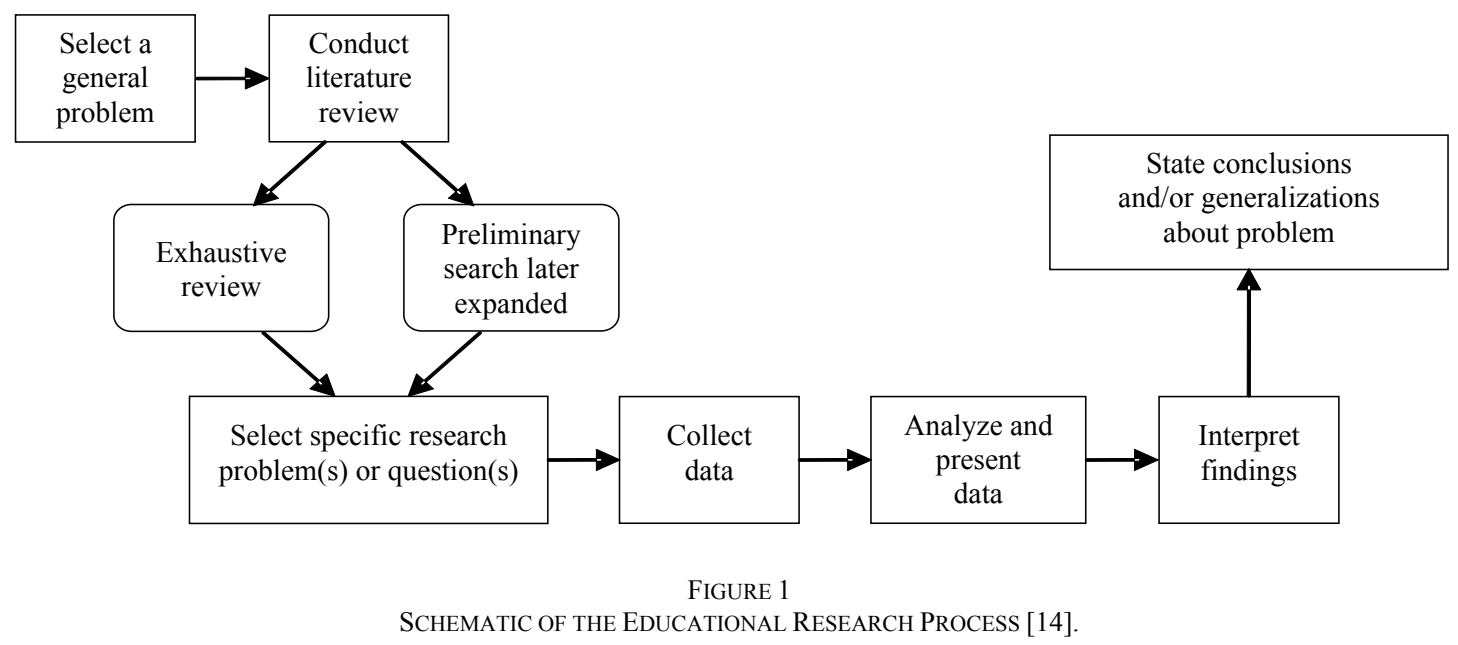




\section{Developing the Research Instruments}

After the research questions have been carefully defined, the instruments that will be used to collect data and to generate multiple perspectives on the questions may be developed [24]. There are a variety of trusted approaches to conducting research in education, and Olds, Moskal, and Miller [17] describe the characteristics, benefits, and drawbacks of several. Each method has its own limitations, and the appropriate methods must be carefully selected to best address the questions at hand. An advantage to using multiple methods within a single study is that the credibility of a hypothesis will be enhanced greatly if it can withstand scrutiny by multiple methods [15].

In addition to selecting the research methods, the instruments themselves should be designed to satisfy two basic principles for measurement: test validity and test reliability $[10,14]$. Test validity describes how relevant the instrument is to the research questions and to what extent conclusions can be drawn. Validity of an instrument can be enhanced by soliciting expert evaluation of instrument items, by pre-testing the instrument and conducting follow-up focus groups, and by using parts of previously validated instruments. Test reliability refers to the consistency of the instrument with respect to time and with respect to different versions of the same instrument. Test reliability can be estimated using a test/retest approach by examining the scores of an individual on two separate occasions for the same instrument. Both validity and reliability are necessary characteristics to draw meaningful conclusions regarding the research questions, and to the extent possible, all qualitative and quantitative instruments should be designed to maximize validity and reliability of the measures used.

PACES Research-A Case Study: For the PACES-2 project, the PACES Team developed a comprehensive, twopart survey instrument to investigate the research questions. The team chose to use a survey (rather than other research instruments such as interviews or observation) for several reasons: a survey is a self-reporting instrument that is easy to administer; it can be conducted easily online or scanned; it can maintain anonymity of subjects; items can be framed to elicit specific responses; and it is an efficient method for collecting large amounts of data in short time periods. However, the team realized that creating a valid, reliable survey is time intensive and that careful thought must be given to every aspect of the survey-development process, including directions that accompany the instrument, the wording and ordering of the items, and testing of the instrument $[17,25]$. As such, the team devoted several months to designing the research instruments and testing the survey prior to broadly administering it.

The two-part survey instrument included the PACES-2 Survey and the Defining Issues Test 2 (DIT-2). The PACES-2 Survey was developed especially for the project, and it included items to measure the variables of the theoretical model. In addition, several separate items were designed to measure each variable so that data could be collected in multiple ways and analyzed for consistency. Consistent with the research questions, the PACES-2 Survey also included items about cheating in college, discipline of study, class level, and demographics. Also, to control for social desirability bias and validate self reports about cheating, the Balanced Inventory of Desirable Responding instrument was included verbatim at the end of the PACES-2 Survey [18]. A prototype of the PACES-2 Survey was delivered to a university office for examinations several weeks prior to the scheduled administration of the survey to allow for conversion of the prototype into a machine-readable form. The same office printed the forms, scanned the data, and stored results in electronic files.

The second part of the instrument, the DIT-2, was chosen because it is a validated instrument that measures the additional moral component of the theoretical model and because it could be used to triangulate data from the PACES-2 Survey. Further, it has been shown to produce good internal and test/retest reliability and has shown discriminate validity from intelligence and political views. Therefore, the PACES Team used an existing instrument with a proven track record (rather than designing a new instrument) to improve validity. The DIT-2 is a multiple-choice test originally developed by Rest $[21,22]$ and purchased in advance from the Center for the Study of Ethical Development at the University of Minnesota [21].

\section{Identifying and Recruiting Subjects}

Identifying a subject pool can be a challenge for several reasons. First, obtaining contact information for a population may require persistent contact with university administrators and can be difficult at a large institution. Second, approval for research involving human subjects requires that any individual in the available population has a reasonable chance of being in the sample [10], so some methodology for random selection must be used. Third, researchers can carefully control the applicable pool of subjects, but they often cannot ensure adequate response rate [17]. Instead, a certain response rate may be targeted and can be achieved by randomly sampling the nonrespondents to achieve the higher response rate [4]. Another way to increase the response rate is to offer incentives, such as cash or gifts. Monetary incentives have been shown to have significant positive effects on response rates of mail surveys $[23,26]$, and a larger incentive has been shown to yield higher response rates $[8,26]$. However, unless cash incentives are used, paperwork may be required to permit taxes to be withdrawn for university students employed by the university.

PACES Research-A Case Study: To identify eligible candidates, the PACES Team obtained enrollment lists from the registrar. However, because the information that was to be collected was potentially embarrassing (i.e., the level of college cheating), the politics involved with obtaining the enrollment lists proved to be difficult in some instances and the process took much longer than anticipated. After the lists were finalized, subjects were randomly selected and were 
contacted by letter (printed on official university letterhead) two weeks before the survey administration. The letter of invitation described the research study and the intended use of the results and included a map to the survey administration site. Also, the PACES Team offered a cash incentive of \$20 for all subjects (made possible through outside funding) to further increase the response rate, and this was described in the letter of invitation. The subjects were reminded of the survey by email one week before the survey.

\section{Seeking Approval for Human Subjects' Research}

To ensure that human subjects involved in the research are protected from risk, approval for the research plan from an Institutional Review Board (IRB) is required. In general, the IRB is a group of individuals who are authorized to determine whether research studies by colleagues affiliated with the institution comply with institutional regulations, professional standards of conduct and practice, and the human subjects' provisions of the Code of Federal Regulation for the Protection of Human Subjects [11]. The Behavioral Sciences (or equivalent) branch of the IRB protects the rights and welfare of human research subjects recruited to participate in research activities that encompass social, behavioral, and educational research and that are considered medically noninvasive. This is the appropriate branch for most engineering education research studies.

The IRB application typically involves specifying information about key personnel and sites at which the research will be performed, identifying any conflicts of interest, and describing the methods for recruiting and compensating study subjects. In addition, any risks to participating subjects must be described, and details about obtaining informed consent and maintaining subject confidentiality are required. Besides clearly defining the research design and the protocol for administering the survey, the instruments themselves must all be submitted.

The time required to navigate the IRB approval process can vary greatly, but a decision may take several weeks. Since any data collected prior to approval may not be used for research purposes, the process should be initiated well in advance of the data collection period of the project. Further, any later changes or modifications to the research design, instruments, or informed consent documents require resubmission to IRB before they may be used.

PACES Research-A Case Study: For this project, the PACES Team applied for IRB approval eight weeks prior to the scheduled start of data collection. The application was completed online, and both parts of the survey instrument were submitted electronically (PACES-2 and DIT-2), as were the letter of invitation and the text of the email reminder note. The consent form to be used at the survey administration included instructions for the instrument, a description of the research study, a statement of the subject's right to confidentiality, a statement of the voluntary nature of the study, the informed consent statement and check box, and contact information for the researchers. This document was also submitted with the IRB application. Four weeks after the initial application was submitted, the team received notification that additional information was requested. In particular, because of low anticipated response rates but because it was possible that a high number of subjects might attend, the PACES Team originally included a statement that the number of students eligible to receive the cash incentive was limited (because of funding limitations). However, the IRB required all students who showed up to be eligible for the incentive. Also, lengthy discussions about the tradeoff of obtaining informed consent with a subject's signature (versus a check box) and maintaining subject anonymity ensued. Finally, it was agreed that a simple checkbox on the consent form (with no identifying information) would suffice. After several iterations and six weeks after the application was initially submitted, the application was approved.

\section{Testing The Survey}

Before collecting data in a full-scale manner, the survey was tested. This included developing a systematic process for administering the survey, testing the protocols and the twopart survey instrument for clarity, assessing the response rate, and establishing the temporal stability and internal consistency of the newly-designed PACES-2 Survey.

\section{Survey Logistics}

The PACES Team spent considerable time carefully planning logistics for administering the survey so that all procedures for human subjects' research were followed and so thorough records were kept. First, to maximize response rate, two separate one-hour sessions were scheduled for the survey, and subjects were invited to attend whichever of the two sessions was most convenient. A cash incentive of \$20 was offered to further increase the response rate.

A list of all subjects invited to participate in the survey was printed, and when subjects arrived for the survey their identification was checked to ensure that they were invited to participate. The list allowed the team to verify that the number of surveys collected at any given time was consistent with the number of students who had arrived and to monitor the overall response rate over time. Subjects were given a packet of information that included two duplicate consent forms (one for the PACES Team and one for the subject) and the two-part survey instrument. The consent forms and the surveys were coded to match so that surveys from subjects who did not provide their consent could be removed from the study. The project team explained the directions, focusing especially on the informed consent document (explaining that all subjects would be compensated for their participation regardless of whether or not they marked the consent box), the voluntary nature of participation, and subject privacy. The subjects were then escorted to a nearby classroom and asked to complete the survey in an unsupervised way. Subjects were allowed one hour to complete the surveys. 
When subjects finished the survey, the team requested a verbal confirmation that each subject had read and understood the consent document (though not whether they actually consented). Then, subjects were asked to place the consent document in one sealed box, the PACES-2 Survey in another, and the DIT-2 Survey in a third. Finally, each subject was compensated with $\$ 20$ in cash, regardless of the time they took to complete the survey and regardless of whether or not they consented to allow their data to be used for research purposes.

\section{Pilot Testing}

It was important to pilot test the PACES-2 Survey for clarity and ease of completion, to eliminate unnecessary or invalid items on the survey using statistical analysis, and to assess the overall survey logistics. For this purpose, a limited number of students (i.e., 120 students) were invited to complete a pilot version of the PACES-2 Survey, and open-ended questions were included so that subjects could comment on the language of both the instructions and of individual items. Subjects were asked to arrive promptly at the start time for either of two separate one-hour sessions during which they could complete the survey and earn $\$ 20$ in cash. The team predicted a $25 \%$ response rate and, because theoretically $100 \%$ of the invited subjects could attend, they printed surveys and had cash-onhand for all 120 invited subjects.

The response rate for the pilot test was extremely lowonly 14 of the 120 students invited actually completed the survey. To potentially increase the response rate for later administrations of the survey, the PACES Team adjusted the survey protocol by extending the session length to three hours and using a rolling start time (i.e., subjects were invited to come at any time during the three hour session ). Because the response rate was too low to allow statistical analysis of the questions on the survey, the instrument itself was not refined. However, the wording for several items on the survey was changed in response to comments regarding readability. Most suggestions resulted in straightforward clarifications of specific questions, but some subjects commented on the repetition of the questions (purposefully designed to test for consistency) and some noted that the items probing about "cheating" in general (with no definition of the term) were confusing. In response to these suggestions, the PACES Team included a statement requesting subjects to use their own definition of what constitutes cheating.

\section{Establishing Temporal Stability and Internal Consistency}

The PACES-2 Survey was subjected to a test/retest reliability study to establish temporal stability and internal consistency of the instrument. Subjects were invited to complete the survey a first time and, if they did, to complete the survey a second time two weeks later. Although the change in format for administering the survey (from two separate one-hour sessions with a specified start time to two separate three hour sessions with a rolling start time) was intended to increase the

\section{0-7803-9077-6/05/\$20.00 @ 2005 IEEE}

response rate, a total of 300 subjects were randomly selected with the hopes that at least 30 of them (consistent with the experimental response rate from the pilot test) would complete the survey the first time and 15 of them would complete the second administration of the survey (sufficient to allow statistical analysis of the data). A different population from the one involved in the full research study was identified for the test/retest study to avoid potential cross-contamination of the sample while providing useable data. At each administration of the survey, subjects were given a cash incentive of $\$ 20$.

Because of the time and expense involved in sending paper invitation letters through the U.S. Postal Service, especially for large-scale studies, the PACES Team conducted a mini-experiment during the test/retest study to identify the difference in response rate for subjects invited by paper letter and those invited by electronic mail (hoping to justify a change in protocol so that only electronic correspondence could be used). The team sent paper invitation letters to 150 of the subjects and email invitations to the other 150. Email reminders were sent one week later to both groups.

For the first administration of the test, 46 of the 150 (31\%) subjects who were invited to participate in the survey via a paper copy of the letter completed the survey, and 24 $(16 \%)$ of those who received an initial email invitation participated. Overall, 70 students were eligible to take the test during the second administration of the survey (an overall response rate of $23.3 \%$ ), and they were all informed by email about the details of the retest. A total of 58 of the 70 invited students completed the second survey $(83 \%$ of the eligible population). Because of these results, the PACES Team maintained the protocol of contacting all eligible subjects by U.S. mail followed by an email reminder.

Data from the two administrations of the survey were analyzed for both temporal stability and internal consistency. Over the two week interim, results remained sufficiently stable - the correlation between subjects' responses on the first administration of the survey and their corresponding responses on the second administration was greater than 0.50 for most items on the survey. However, two items showed poor temporal stability, and these items were eliminated from the final survey ${ }^{\nabla}$. To further refine the survey instrument, a reliability analysis was performed on several related items on the survey to identify the most reliable and consistent items. In particular, the PACES Team completed a factor analysis on 20 pairs of semantic differentials used to describe students' attitudes towards cheating. One factor (composed of five data pairs) had the highest consistency among its observed variables (i.e., highest Cronbach's alpha score) and thus provided the highest reliability in measuring the attitudes of the subjects. As such, the five items were kept for the final survey and the remaining 15 pairs were removed from the instrument.

\footnotetext{
$\nabla$ Because of logistical difficulties, the items with low stability were not actually eliminated from the survey instrument. However, the items were excluded from final data analysis.
}

October 19-22, 2005, Indianapolis, IN 


\section{Administering the FinAl SurVey}

After the survey instrument was pilot tested and subjected to a test/retest analysis, it was administered broadly. A total of 800 subjects were invited to participate in the full administration of the survey, and a response rate of $25 \%$ was anticipated. Again, subjects were randomly selected from enrollment lists (excluding those who previously were invited to participate). Paper letters of invitation were mailed through the U.S. Postal Service two weeks before the scheduled administration of the survey, and email reminders were sent one week later. Two separate sessions, each three hours in length, were offered, and subjects were invited to arrive at any time during the session of their choice. As before, all subjects were given a cash incentive of $\$ 20$ for completing the survey.

A total of 228 subjects completed the survey (response rate greater than $27 \%$ ), and 222 consented to allow their data to be used for research purposes. Survey administration went smoothly.

\section{DISCUSSION}

This paper concerns the design, testing, and administration of the PACES-2 Survey, and a full report on the results of the survey are the subject of a future article. However, the experiences described here, particularly the authors' approaches to establishing a team of qualified researchers, managing response rate, and seeking approval for human subjects' research, can be applied to other settings.

The PACES Team (Finelli, Carpenter, and Harding) are engineering educators with no formal training in education research. However, the team has collected a library of resources about research in engineering education, has improved their quantitative analysis skills and acquired proficiency in qualitative research by participating in workshops and seminars, and has relied on the expertise of others (especially faculty members and graduate students from the field of education) in planning and conducting research in engineering education. This approach to establishing a team of qualified researchers has been a successful one.

The PACES Team has also been successful in managing response rate, a task that is not always easy in survey research. Not only is identifying a suitable pool of subjects and obtaining contact information for them often difficult, but predicting the number of subjects who are likely to participate in the research is problematic. For this project, the PACES Team conducted a pilot test of the survey to estimate the response rate, adjusted the protocol for administering the survey to increase the rate, and experimented with paper versus electronic invitations to participate. Also, the team offered cash incentives to improve the response rate and balanced this with the funding available to compensate the subjects. In the end, the response rate was more than $27 \%$ and was slightly higher than what the research team predicted.

Finally, the research team was successful in navigating the process for approval of research involving human subjects. Two of the institutions involved in the research project do not have an Institutional Review Board, and the one that did have an IRB required official letters from the other institutions, so the process was not straight forward. In addition, the IRBimposed requirement of having subjects sign their name on the informed consent document was problematic in that it would breach the confidentiality. A solution was reached whereby matching code numbers on consent forms and surveys were sufficient.

\section{ACKNOWLEDGMents}

The authors gratefully acknowledge the financial support of the Kern Family Foundation and the Templeton Foundation. The authors would also like to thank Dr. Matthew J. Mayhew, Director of Student Life Assessment at the University of North Carolina at Wilmington for his input on the development of survey items and protocols.

\section{REFERENCES}

1. Ajzen, I. "The Theory of Planned Behavior." Organizational Behavior and Human Decision Processes, 50, 179-211, 1991.

2. Armitage, C.J., and M. Conner. "Predictive validity of the theory of planned behaviour: The role of questionnaire format and social desirability." Journal of Community and Applied Social Psychology, 9: 261-272, 1999.

3. Armstrong, J.S. and T.S. Overton. "Estimating nonresponse bias in mail surveys.” Journal of Marketing Research, 14, 1977.

4. Baird, J.S. "Current trends in college cheating." Psychology in the Schools, 17(4): 515-522, 1980,

5. Beck, L., and I. Ajzen. "Predicting dishonest actions using the Theory of Planned Behavior." Journal of Research in Personality, 25(3): 285-301, 1991.

6. Bowers, W.J. Student Dishonesty and its Control in College. Bureau of Applied Social Research, Columbia University, New York, NY. 1964.

7. Brown, B.S. "A comparison of the academic ethics of graduate business, education and engineering students." College Student Journal, 30(Sept. 1996): 294-301, 1996.

8. Church, A.H. "The effect of incentives on mail survey response rates: A meta-analysis." Public Opinion Quarterly, 57(1), 1993.

9. Creswell, J.W. Qualitative Inquiry and Research Design. Sage Publications, Thousand Oaks, CA. 1998.

10. Gall, M.D., J.P. Gall, and W.R. Borg. Educational Research: An Introduction (Seventh Edition). Pearson Education, Inc., Boston, MA. 2003.

11. Harp, J., and P. Taietz. "Academic integrity and social structure: A study of cheating among college students." Social Problems, 13(4): 365$373,1996$.

12. Jackson, C.J., S.Z. Levine, A. Furnham, and N. Burr. "Predictors of cheating behavior at a university: A lesson from the psychology of work.” Journal of Applied Social Psychology, 32(5): 1031-1046, 2002.

13. McCabe, D.L. "Classroom cheating among natural science and engineering majors." Science and Engineering Ethics, 3, 433-445, 1997.

14. McMillan, J.H. and S. Schumacher. Research in Education (Fourth Edition). Addison-Wesley Educational Publishers, Inc., New York. 1997. 
15. National Research Council. Scientific Research in Education. Shavelson, R.J. and L. Towne, eds. National Academy Press, Washington, DC. 2002

16. Newstead, S.E., A. Franklyn-Stokes, and P. Armstead. "Individual differences in student cheating." Journal of Educational Psychology, 88(2): 229-241, 1996

17. Olds, B.M., B.M. Moskal, and R.L. Miller. "Assessment in engineering education: Evolution, approaches, and future collaborations." Journal of Engineering Education, 94(1): 13-25, 2005.

18. Paulhus, D.L. "Measurement and control of response bias." In Measures of Personality and Social Psychological Attitudes, Robinson, J.P. and L.S. Wrightsman, eds. Academic Press, Inc., San Diego, CA. 1991.
19. Radcliff, D.F., and L. Jolly. "Dilemmas in framing research studies in engineering education." Proceedings of 2003 ASEE Annual Conference and Exposition, 2003.

20. Rawwas, M.Y.A., and H.R. Isakson. "Ethics of tomorrow's business managers: the influence of personal beliefs and values, individual characteristics, and situational factors." Journal of Education for Business, 27, 2000.

21. Rest, J.R., and D. Narvaez. Guide for DIT-2. Center for the Study of Ethical Development, University of Minnesota. Minneapolis, MN. 1998.

22. Rest, J.R., D. Narvaez, J. Thomas, and M.J. Bebeau. "DIT2: Devising and testing a revised instrument of moral judgment." Journal of Educational Psychology, 89(1): 5-28, 1999.

23. Singer, E., J. Van Hoewyk, N. Gebler, T. Raghunathan, and K. McGonagle. "The effect of incentives on response rates in interviewermediated surveys." Journal of Official Statistics, 15(2), 1999.

24. Steffe, L. and P.W. Thompson. "Teaching experimental methodology: Underlying principles and essential elements." In Handbook of Research Design in Math and Science Education, Kelly, A.E. and R.A. Lesh, eds. 2000.

25. Suskie, L.A. Questionnaire Survey Research: What works? (Second Edition). Florida State University: Association for Institutional Research, 1996.

26. $\mathrm{Yu}, \mathrm{J}$. and $\mathrm{H}$. Cooper. "A quantitative review of research design effects on response rates to questionnaires." Journal of Market Research, 20, 1983. 